\title{
Habilidades cognitivas e uso de benzodiazepínicos em idosas institucionalizadas
}

\author{
Cognitive skills and the use of benzodiazepinic \\ drugs in institutionalized elderly women \\ Mônica Giaretton Bicca', Irani Iracema de Lima Argimon
}

\section{RESUMO}

Objetivo: O objetivo desta pesquisa foi verificar as habilidades cognitivas em idosas usuárias de benzodiazepínicos e as idosas não-usuárias deste tipo de medicação. Participaram do estudo 123 idosas institucionalizadas. Método: $O$ delineamento foi de um estudo quantitativo e de um transversal. Os instrumentos utilizados foram: ficha de dados sociodemográficos, breve entrevista internacional de neuropsiquiatria modificada, mini-exame do estado mental, escala de depressão geriátrica, span de números, teste de fluência verbal (categoria animal) e teste de evocação seletiva livre e com pistas (Buschke). Resultados: A idade variou entre 60 e 101 anos, com a média de 79,73 anos (DP =9,56). Não houve diferença em relação à idade ou aos anos em que residência na instituição asilar $(p=0,846)$. $O$ tempo mínimo foi de 1 ano e o máximo, de 26 anos, a média ficou em 4,02 anos (DP = 4,38).

\section{Palavras-chave}

Habilidades cognitivas, benzodiazepínicos, idosas.

\section{Keywords}

Cognitive skills, benzodiazepinic, elderly women.
O tempo médio de estudo foi de 5,98 anos ( $\mathrm{DP}=4,17), 80,5 \%$ sabem ler e $79,7 \%$ sabem escrever. Conclusão: Não foram encontradas associações significativas entre as habilidades cognitivas em idosas usuárias de benzodiazepínicos e as não-usuárias.

Objective: The aim of this research is the verification of cognitive skills in elderly women using benzodiazepines and non-users of this medication. The study consisted of 123 institutionalized elderly women. Method: The study was delineated as quantitative and cross-sectional. The instruments used were: sociodemographic data chart, brief international interview of modified neuropsychiatry, mini-exam of the mental state, geriatric depression scale, numbers span, verbal fluency test - category animal, test of selective evocation, free and with leads (Buschke). Results: Age ranged from 60 to 101 years, mean $=79.73$ years $(S D=9.56)$. There was no difference related to age or years spent in institutions $(p=0.846)$. Minimum period of time was one year and maximum duration was 26 years, mean $=4.02$ years $(S D=4.38)$. The average follow- $u p$ time was 5.98 years (SD $=4.17), 80.5 \%$ of the individuals could read and $79.7 \%$ could write." Conclusion: No significant associations were found between the cognitive skills in benzodiazepinic using elderly women and the ones who do not use this medication. 


\section{INTRODUÇÃO}

A Organização Mundial da Saúde (OMS)' sugere 65 anos como marcador do início da terceira idade em países desenvolvidos e 60 anos, para países em desenvolvimento. Esta é considerada uma fase normal do ciclo vital, vista como processo biológico natural, não-patológico, caracterizado por uma série de alterações morfofisiológicas, bioquímicas e psicológicas que ocorrem no organismo ao longo da vida. Sabe-se que o envelhecimento biológico é o processo que antecede alguns limites. Na realidade, a chamada terceira idade apresenta características biopsicossociais, com efeitos que incidem sobre as pessoas e a sociedade de maneira especial².

Para Vieira³, cognição é o termo global empregado para descrever as habilidades cognitivas ou o funcionamento mental que implica a habilidade para sentir, pensar, perceber, lembrar, raciocinar, formar estruturas complexas de pensamento e a capacidade para produzir respostas às solicitações e aos estímulos externos.

Usualmente, o envelhecimento está associado à lentidão de raciocínio e às dificuldades de memória, assim acredita-se que os idosos têm dificuldades em compreender e lembrar situações novas ou que lhes são apresentadas rapidamente. Ainda há desinformação ou desconhecimento a respeito do que é envelhecimento cognitivo normal e como distingui-lo do envelhecimento patológico 4 .

Aliado a este fenômeno biopsicossocial que afeta tanto o idoso, as limitações cognitivas podem ser potencializadas e/ou aceleradas pelo uso de benzodiazepínicos. Os benzodiazepínicos estão entre os medicamentos mais utilizados no mundo, estima-se que, em 2001, foram 6,96 bilhões de doses diárias como hipnóticos consumidos no mundo, número bastante significativo $0^{5-7}$.

A dependência de benzodiazepínicos pode desenvolver-se em dias ou semanas, visto que os principais sintomas de descontinuação do uso são opostos ao efeito terapêutico esperado - alívio da insônia, depressão, ansiedade e dor - ou são intensificação da recorrência dos sintomas originais ${ }^{8-11}$.

Pesquisas epidemiológicas indicam que a prática do uso de benzodiazepínicos difere bastante em relação a eficácia e a efetividade, bem como das recomendações internacionalmente aceitas. Eles estão entre os medicamentos mais prescritos a idosos, porquanto as mulheres os utilizam em proporção duas vezes maior do que os homens. Fundamentando-se nesta idéia, Huf, Lopes e Rozenfeld ${ }^{12}$ avaliaram o padrão de uso desta medicação em um centro de convivência de mulheres com mais de 60 anos. Foi utilizado um questionário-padrão, com perguntas fechadas e testado por meio de estudo-piloto (não incluídos na análise). A prevalência do uso naquele período foi estimada em
21,3\% e a prevalência de uso diário por 12 meses ou mais foi de $7,4 \%$.

De acordo com Paprocki ${ }^{13}$, o consumo crescente de benzodiazepínicos pode ser resultado de período turbulento, que caracteriza as últimas décadas da humanidade, quais sejam, a diminuição da tolerância ao estresse, a introdução de novas drogas e, ainda, os hábitos de prescrição inadequada por parte dos médicos. No caso de idosos isso também pode ser observado e o problema é exacerbado em virtude de não estarem conscientes dos riscos agregados a estas drogas, o que os leva a ignorar o fato de os benzodiazepínicos estarem associados à possível deterioração da atenção e das capacidades cognitivas ${ }^{14}$.

Em um estudo recente, Auchewski et al. ${ }^{15}$ apresentaram os principais efeitos colaterais que comprometem o usuário de benzodiazepínicos, como diminuição da atividade psicomotora, prejuízo na memória, desenvolvimento de dependência e a qualidade da orientação médica sobre os efeitos colaterais. Foram entrevistados 120 pacientes com mais de 48 anos que procuraram esta medicação em farmácias dos principais centros do país. Os resultados obtidos, por meio de questionário de perguntas abertas, foram os seguintes: 13\% dos entrevistados relataram que foram orientados sobre os três tipos principais de efeitos colaterais, 27\%, a respeito de pelo menos dois, e, 40\%, sobre pelo menos um, enquanto, 19\%, não receberam nenhuma orientação.

Tendo em vista a relevância do tema, a presente pesquisa teve como objetivo geral identificar as habilidades cognitivas em idosas dependentes de benzodiazepínicos e as não-dependentes. Como objetivos específicos, pretendeuse avaliar as habilidades cognitivas em idosas usuárias de benzodiazepínicos e as não-usuárias, no que tange a memória, atenção e fluência verbal neste grupo.

\section{MÉTODO}

\section{Delineamento}

Foi realizado estudo transversal, utilizando-se metodologia quantitativa.

\section{Participantes}

Participaram do estudo 123 idosas institucionalizadas, com idade igual ou superior a 60 anos, de escolaridade e níveis socioeconômicos diversos, residentes em instituições asilares na cidade de Porto Alegre. Como critérios de inclusão, as participantes do grupo 1 deveriam ser usuárias de benzodiazepínicos e as participantes do grupo 2 não poderiam fazer uso de benzodiazepínicos. Foram excluídas do estudo as idosas com presença de transtornos psicológicos graves, como transtornos psicóticos e sintomas graves de depressão. 


\section{Instrumentos}

Para a coleta de dados, foram empregados os seguintes instrumentos: ficha de dados sociodemográficos, breve entrevista internacional de neuropsiquiatria modificada (MINI), mini-exame do estado mental (MMSE), escala de depressão geriátrica (EDS), span de números, teste de fluência verbal (categoria animal), teste de evocação seletiva livre e com pistas $^{16}$, que serão descritos a seguir.

A ficha de dados sociodemográficos consiste de itens incluindo idade, sexo, estado civil, tempo de residência em instituição asilar, anos de escolaridade, profissão, proveniência da renda, religião, atividades de lazer, informações sobre saúde, sobre o uso de benzodiazepínico e dosagem utilizada.

A MINI, versão empregada em mais de 30 idiomas, validada no Brasil por Amorim7, utilizada para verificar os critérios de exclusão, consiste de breve entrevista estruturada para a triagem dos principais quadros psicopatológicos. Permite a formulação de diagnósticos compatíveis com os critérios do DSM-IV (Manual Diagnóstico e Estatístico dos Transtornos Mentais - APA) e da CID-10 (Classificação Internacional de Doenças - OMS).

O MMSE de Folstein, Folstein e McHugh ${ }^{18}$ foi utilizado para fazer o rastreamento inicial do estado mental e avaliar a presença de déficits cognitivos. Ele é composto por questões subdivididas em sete categorias, organizadas de maneira que avalie funções cognitivas específicas: orientação para tempo (5 pontos), orientação para local (5 pontos), registro de três palavras (3 pontos), atenção e cálculo (5 pontos), lembrança das três palavras (3 pontos), linguagem (8 pontos) e capacidade construtiva visual (1 ponto). O escore deste teste pode variar de zero (mínimo) até o total de 30 pontos (máximo), com ponto de corte de 24.

A EDS, desenvolvida por Yesavage, Brink e Rose ${ }^{19}$, foi utilizada para identificar os sintomas depressivos das idosas. $O$ instrumento consiste de 15 questões, com alternativas de resposta sim e não, em que escores inferiores a cinco são considerados normais, de cinco a dez indicam depressão leve a moderada e, acima de dez, indicam depressão grave.

A escala de inteligência de adultos de Wechsler III, subteste de span de números ${ }^{20}$, é utilizada para avaliar a atenção. Compreende duas tarefas diferentes de dígitos, na ordem direta e inversa. É empregada para medir memória de trabalho, sendo o escore total o "valor bruto", ou seja, o somatório dos acertos da ordem direta e inversa.

O teste de fluência verbal (categoria animal), de Spreen e Benton, validado por Brucki et al.21, avalia a capacidade de evocar palavras de categoria semântica sob condições dirigidas e o escore corresponde ao número total de nomes de animais produzidos em um minuto.

O teste de evocação seletiva livre e com pistas (Buschke) tem a função de mensurar a memória de retenção, armazenamento e evocação, foi utilizado o proposto por Bus- chke e Fuld ${ }^{16}$, que consiste da apresentação de uma prancha com 16 figuras a serem memorizadas. A participante é orientada a apontar para cada um dos itens, conforme for solicitado, por categoria e dar o nome correspondente. Depois de identificar cada figura, a prancha é retirada e é solicitado que a participante verbalize o nome de todos os itens que consiga lembrar. As lembranças livres e com pistas são então retestadas após o período de 30 minutos, sem a apresentação da prancha. O escore é obtido por meio do número total de estímulos recordados, sendo a pontuação máxima de 96 lembranças.

\section{Procedimentos}

Foi realizado o contato com algumas instituições asilares de Porto Alegre para explicar os objetivos da pesquisa e obter as autorizações necessárias.

Após a aprovação do projeto de pesquisa pelo Comitê de Ética da Pontifícia Universidade Católica do Rio Grande do Sul (PUCRS), foi realizada visita às instituições asilares. Alguns locais dispunham de atividades semanais em grupo, nos quais foram feitos os primeiros contatos. Em outros, as idosas receberam as informações individualmente, e aquelas que concordaram em participar do estudo assinaram o termo de consentimento livre e esclarecido (TCLE).

Os instrumentos foram aplicados individualmente em salas ou outro local reservado na própria instituição. A coleta de dados foi realizada pela pesquisadora e por quatro alunos de graduação previamente treinados.

A análise quantitativa foi efetivada por meio de estatísticas descritivas, com o objetivo de traçar o perfil sociodemográfico e fazer o levantamento das estratégias utilizadas. Foram calculados as médias, o desvio-padrão (DP), as freqüências e os percentuais.

Para testar as hipóteses da pesquisa, foi utilizado os testes Mann-Whitney e o t de Student junto com os resíduos ajustados padronizados. Os dados foram tabulados a partir do programa SPSS 11.5, com o nível de significância de 5\%.

\section{RESULTADOS}

\section{Características sociodemográficas}

Das 123 idosas que constituíram a amostra, a idade variou entre 60 e 101 anos, tendo como média 79,73 anos (DP $=9,56)$. Os dois grupos não demonstraram diferença em relação à idade ou aos anos em que residem na instituição asilar ( $p=0,846)$.

O tempo mínimo de residência na instituição asilar foi de um ano e o máximo de 26 anos. A média ficou em 4,02 anos ( $D P=4,38)$. Quanto ao tipo de instituição, $78,9 \%$ das idosas moram em casas asilares particulares e $21,1 \%$, em instituição pública. 
O tempo médio de estudo entre as participantes foi de 5,98 anos ( $\mathrm{DP}=4,17), 80,5 \%$ sabem ler e $79,7 \%$ sabem escrever.

$\mathrm{Na}$ Tabela 1, encontram-se as freqüências relacionadas à amostra, que é constituída somente por mulheres, porquanto a maioria delas é viúva $(62,2 \%)$, com renda proveniente de aposentadoria (52\%).

Tabela 1. Dados sociodemográficos das idosas.

\begin{tabular}{lcc}
\hline Variáveis & $\mathrm{n}(\mathrm{T}=123)$ & $\%$ \\
\hline Estado civil & 12 & \\
$\quad$ Casada/com companheiro & 25 & 20,3 \\
$\quad$ Solteira & 77 & 62,6 \\
$\quad$ Viúva & 7 & 5,7 \\
$\quad$ Separada & 2 & 1,6 \\
$\quad$ Outro & & \\
Renda provém de & 32 & 26 \\
$\quad$ Pensão & 64 & 52 \\
Aposentadoria & 2 & 1,6 \\
Ajuda de terceiros & 6 & 4,9 \\
Outra & 19 & 15,4 \\
$\quad$ Mais de uma categoria & & \\
Como estáa saúde & 55 & 44,7 \\
$\quad$ Boa & 56 & 45,5 \\
$\quad$ Regular & 12 & 9,8 \\
$\quad$ Má & & \\
Faz exames de rotina & 16 & 13 \\
$\quad$ aão & 16 & 87 \\
Sim & 107 & \\
\hline
\end{tabular}

Observou-se que, de maneira expressiva, as idosas consideram sua saúde, no mínimo, como regular (45,5\%), entre elas, $87 \%$ faz exames médicos rotineiramente.

Verificou-se como resposta positiva "ter uma religião", pois $93,5 \%$ das idosas mencionaram ser de determinada religião. No entanto, quanto à prática efetiva de ir à missa, rezar e manter isso como rotina, este percentual é de 84,6\%.

A principal atividade das idosas foi assistir televisão (69,9\%), seguida de ouvir música $(60,2 \%)$ e atividades manuais (55,3\%).

Tabela 2. Atividades de lazer das idosas.

\begin{tabular}{lcc}
\hline Lazer & $\mathrm{n}(\mathrm{T}=123)$ & $\%$ \\
\hline Atividades mentais (leitura, bingo, cartas, & 84 & 68,3 \\
damas, palavras cruzadas) & 109 & $88,6 \%$ \\
Atividades: TV e rádio (novelas, filmes, música) & 24 & $19,5 \%$ \\
Atividades manuais: crochê, tricô, artesanato & 39 & $31,7 \%$ \\
Atividades físicas: caminhada, ginástica, bocha & 35 & $28,4 \%$ \\
Atividades sociais: receber amigos, baile, grupos & 49 & 39,8 \\
Atividades religiosas & &
\end{tabular}

\section{Quanto ao uso de benzodiazepínicos e habilidades cognitivas}

Considerando a relação existente entre a pontuação alcançada pelas idosas nos testes de memória e o uso de benzodiazepínicos, por meio do teste t de Student, não se encontrou associação significativa ( $p=0,846)$.
Os resultados obtidos mediante o teste t de Student, relacionados ao uso de benzodiazepínicos e os escores dos testes de fluência verbal, também não tiveram associação positiva $(p=0,783)$.

Da mesma forma, não houve associação significativa entre os escores dos testes de atenção e o uso de benzodiazepínicos $(p=0,233)$.

Tabela 3. Comparação das médias das variáveis contínuas entre os grupos que usam e os que não usam benzodiazepínicos.

\begin{tabular}{lcccccc}
\hline & Variáveis & $\mathbf{n}$ & $\mathbf{M}$ & $\mathbf{D P}$ & $\mathbf{t}$ & $\mathbf{p}$ \\
\hline MMSE & Não & 94 & 22,11 & 9,47 & $-0,971$ & 0,334 \\
SOL & Sim & 29 & 23,21 & 9,99 & & \\
\multirow{3}{*}{ Spantotal } & Não & 94 & 50,80 & 18,99 & $-1,423$ & 0,157 \\
& Sim & 29 & 56,62 & 20,14 & & \\
\multirow{3}{*}{ Fluência verbal } & Não & 94 & 7,45 & 3,26 & $-1,199$ & 0,233 \\
& Sim & 29 & 8,28 & 3,21 & & \\
& Não & 94 & 11,0 & 4,78 & $-0,276$ & 0,783 \\
\hline
\end{tabular}

\section{DISCUSSÃO}

A OMS ${ }^{22}$, em um relatório sobre o uso de substâncias psicoativas, considerou o consumo destas substâncias potencialmente danoso à saúde e salientou que o conhecimento dos fatores que influenciam o início e a continuação do uso é incompleto. Este relatório corrobora o estudo de Barker et al. ${ }^{23}$, que versam sobre a ampla prescrição desta medicação e a falta de estudos que avaliem a alteração da habilidade cognitiva depois de muito tempo de uso da substância.

As idosas que participaram deste estudo, em sua maioria, desempenham seu dia-a-dia de modo independente e autônomo, parecem ser clinicamente saudáveis para sua faixa etária e apresentam níveis de escolaridade e renda que correspondem aos índices das camadas médias da população, dados estes semelhantes aos encontrados em um estudo realizado por Dellaroza ${ }^{24}$

No presente estudo, não foi evidenciada associação positiva entre o uso de benzodiazepínicos e a dificuldade nas habilidades cognitivas de memória, atenção e linguagem, o que contraria a idéia de que os idosos usuários desta substância teriam algum tipo de prejuízo. Este resultado vem ao encontro do estudo de Verdoux, Lagnaoui e Begaud ${ }^{25}$, que revisaram trabalhos buscando saber sobre a possibilidade de o uso de benzodiazepínicos ser um fator de risco para a aceleração do declínio cognitivo e/ou demência em idosos. Para isso, usaram os arquivos de busca da MedLine relacionados ao uso desta substância e à mudança da performance cognitiva. Somente sete artigos encaixaramse nestes critérios, porquanto, do total, dois acreditam que haja baixo nível de relação entre as variáveis, dois não admitem associação entre as categorias e três referem que ha- 
veria aumento da probabilidade de aceleração no declínio cognitivo aliado ao uso de benzodiazepínicos. Os autores acreditam que a discrepância das idéias se dê, possivelmente, por questões metodológicas, especialmente em relação à definição de cognição e de exposição à droga. Ainda, em razão de grande parte da população fazer uso indiscriminado deste psicotrópico, fica difícil mensurar as conseqüências para a saúde da população.

No que se refere às habilidades cognitivas, deve-se salientar que houve decréscimo na memória de trabalho. As idosas tiveram desempenho abaixo do esperado e não conseguiram aproveitar as pistas. Este indício também é referido por Xavier et al. ${ }^{26}$, que consideram a perda da memória como a primeira habilidade cognitiva a deteriorar. Argimon ${ }^{27}$ salienta que ainda não está definido na literatura o quanto o declínio da memória é apenas uma forma de envelhecimento cognitivo usual ou uma patologia que não pode ser vista como variante mais grave da normalidade.

No MMSE, as idosas conservaram-se abaixo do ponto de corte que diferencia indivíduos com prejuízo cognitivo. Entretanto, quando comparados o desempenho no teste, a idade e os anos de escolaridade, elas conseguem ter bom aproveitamento.

Lesak $^{28}$ acredita que as mudanças cognitivas ao longo do envelhecimento ocorrem nas esferas sensório-motoras, visoespaciais, de atenção, memória e linguagem, sucessivamente. Na presente pesquisa, um aspecto evidenciado foi o bom desempenho nos escores do teste de fluência verbal, indicando que, para muitas idosas, a linguagem ainda está preservada e em pleno funcionamento.

Sabe-se que diferentes variáveis entram em jogo quando são avaliadas as habilidades cognitivas da população idosa, quais sejam, idade, escolaridade, estilo de vida, relacionamento interpessoal, atividades físicas, entre outras, as quais contribuirão para que tal população possa estar mais saudável27. Outra variável que merece consideração é a de que, uma vez institucionalizado, o idoso recebe cuidados diuturnamente, que podem prevenir problemas e dificuldades, que muitas vezes só seriam diagnosticados em fase avançada. Esta variável também pode ser observada em um estudo feito por Oliveira et al. ${ }^{29}$ que mostra a qualidade do serviço oferecido por algumas instituições asilares.

É importante ressaltar que a maioria das participantes provém de instituições privadas, levando a pensar que adequadas condições de uso e monitoramento da medicação podem funcionar como fator de proteção das habilidades cognitivas destas idosas. Também, de acordo com as características sociodemográficas das participantes, o envolvimento com atividades de lazer e atividades físicas pode estar relacionado com fatores de proteção das habilidades cognitivas destas idosas, mesmo fazendo uso de medicação. Estas idosas encontram várias opções de entretenimento, como cinema, teatro, aulas de artesanato, missas, bingo e, principalmente, atividades que exercitam a mente. Este fator é amplamente discutido por Argimon e $\operatorname{Stein}^{30}$, que consideram as atividades mentais como protetivas ao declínio cognitivo.

Observou-se que há idosos mais jovens em instituições, dessa forma, percebe-se que a institucionalização nem sempre é vista como fator ruim, já que se sentem amplamente cuidadas e acolhidas. Algumas dizem preferir estar sob o cuidado de uma equipe, porque estão sendo assistidas em tempo integral, além de ficarem tranqüilas em relação à sua saúde e aos cuidados médicos; ainda, há aquelas que conseguem reproduzir parte de suas casas dentro do asilo.

Uma das limitações deste estudo foi a dificuldade de encontrar informações precisas quanto ao uso da medicação, especialmente no que se refere ao tempo de uso. Grande parte das idosas entrevistadas não soube informar desde quando utiliza benzodiazepínicos, informação esta que também não foi encontrada nos prontuários médicos. Esta limitação também foi encontrada por Linden et al. ${ }^{31} \mathrm{em}$ um estudo sobre a prevalência do uso de benzodiazepínicos, em que as participantes não conseguiram dar informações mais consistentes sobre essa prática.

Sabe-se que o uso continuado de benzodiazepínicos dificilmente encontra indicação farmacológica, principalmente porque pode causar tolerância à medicação e dependência. Além do que, embora sejam drogas relativamente seguras, têm tido restrições em sua utilização cada vez maiores, por causa da incidência de seus efeitos colaterais ${ }^{32}$.

Poyares et al. ${ }^{33}$ sugerem que o uso de benzodiazepínicos em idosos deve ser racional, uma vez que há indícios, por exemplo, do aumento do risco de mortalidade com seu uso crônico, e deveriam ser avaliados o padrão e as razões de uso, dos efeitos do uso continuado e das estratégias utilizadas pelos clínicos e usuários para cessar seu uso. No entanto, a idade, no presente estudo, que é preditor importante do uso prolongado de benzodiazepínicos, parece ter sua importância diminuída quando a análise se restringe aos maiores de 60 anos, não tendo influência em nenhuma das variáveis.

Cabe destacar que, por se tratar de idosas institucionalizadas na cidade de Porto Alegre, os achados deste estudo não podem ser generalizados.

\section{CONCLUSÃO}

A partir dos resultados deste estudo, não foi possível encontrar associação entre as habilidades cognitivas em idosas usuárias e não-usuárias de benzodiazepínicos. Há de se considerar, ainda, a falta de informações precisas sobre o uso dos benzodiazepínicos, principalmente em relação ao tempo de uso que pode ter interferido nos resultados. No entanto, pode-se pensar que, até o momento, são poucos os estudos longitudinais que avaliam os fatores relacionados 
ao uso prolongado de benzodiazepínicos, e os estudos transversais apresentam grandes limitações neste sentido. Os achados desta pesquisa apontam a necessidade de trabalhos em que os pacientes que iniciam o uso de benzodiazepínicos possam ser acompanhados ao longo do tempo, permitindo explorar melhor os fatores envolvidos.

\section{REFERÊNCIAS}

1. OMS. Organización Mundial de la Salud. Aplicaciones de la epidemiologia al estudio de los ancianos: informe de un grupo cientifico de la OMS sobre a epidemiologia del envejecimento. Genebra: OMS, Série de informes técnicos. 1984

2. Busse EW, Blazer D. Geriatric psychiatric. Washington: American Psychiatric Press. 1989.

3. Vieira EB. Manual de gerontologia: um guia teórico-prático para profissionais, cuidadores e familiares. Rio de Janeiro: Revinter. 1996.

4. Parente MA, Taussik I. Neuropsicologia: distúrbios de memória e esquecimentos benignos. 2002 [acesso em 2005 Jul 22]. Disponível em: Recuperado em 22 jul. 2005: http://www. comciencia.br.

5. Higgins K, Cooper-Stanbury M, Williams P. Statistics on drug use in Australia in 1998. Canberra: Australian Institute of Health and Welfare. 2000.

6. Vicens C, Fiol F, Llobera J, Campoamor F, Mateu C, Alegret S, et al. Withdrawal from longterm benzodiazepine use: randomised trial in family practice. (2006). Br J Gen Pract. 2006;56:958-63.

7. Nurmi-Lüthje I, Kaukonen JP, Lüthje P, Naboulsi H, Tanninen S, Kataja M, et al. Use of benzodiazepines and benzodiazepine-related drugs among 223 patients with an acute hip fracture in Finland: comparison of benzodiazepine findings in medical records and laboratory assays. Drugs Aging. 2006;23(1):27-37.

8. Poyares D, Guilleminault C, Ohayon MM, Tufik S. Chronic benzodiazepine usage and withdrawal in insomnia patients. J Psychiatric Res. 2004;38(3):327-34.

9. Freitas et al., 2002

10. Boeuf 0, Lapeyre-Mestre M. Survey of forged prescriptions to investigate risk of psychoactive medications abuse in France: results of OSIAP survey. Drug Saf. 2007;30:265-76.

11. Nomura K, Nakao M, Sato M, Yano E. Regular prescriptions for benzodiazepines: a cross-sectional study of outpatients at a university hospital (2006). Intern Med. 2006; 45:1279-83.

12. Huf G, Lopes C, Rozenfeld S. 0 uso prolongado de benzodiazepínicos em mulheres de um centro de convivência para idosos. Cad Saude Publica. 2000;16:351-62.

13. Paprocki J. 0 emprego de ansiolíticos benzodiazepínicos pelo clínico geral e por especialistas não psiquiatras. Rev ABP-APAL. 1990;64:305-12.
14. Stuart-Hamilton I. A psicologia do envelhecimento: uma introdução. Porto Alegre: Artmed. 2002.

15. Auchewski L, Andreatini R, Galduróz J, Lacerda R. Avaliação da orientação médica sobre os efeitos colaterais de benzodiazepínicos. Rev Bras Psiquiatr. 2004;26:24-31.

16. Buschke $H$, Fuld PA. Evolution of storage, retention and retriving in disordered memory and learning. Neurology. 1974;11:1019-25.

17. Amorim P. Mini International Neuropsychiatric Interview (MINI): validação de entrevista breve para diagnóstico de transtornos mentais. Rev Bras Psiquiatria. 2000;3:106-15.

18. Folstein MF, Folstein SE, McHhugh PR. "Mini-mental state": a pratical method for grading the cognitive state of patients for the clinician. J Psych Res. 1975;12:189-98.

19. Yesavage J, Brink T, Rose T. Development and validation of a geriatric depression screening scale: a preliminary report. J Psychiatr Res. 1983;17:37-49.

20. WAIS III. Escala de inteligência Wechsler para adultos: manual, adaptação e padronização de uma amostra brasileira por Elisabeth do Nascimento. São Paulo: Casa do Psicólogo. 2004.

21. Brucki SM, Malheiros S, Okamoto I, Bertolucci P. Dados normativos para 0 uso do teste de fluência verbal (categoria animal), em nosso meio. Arq Neuropsiquiatr. 1997;55:56-61.

22. WHO. World Health Organization. (1998). Drug dependence. Thirtieth Report of WHO EXpert Committee. Geneva: WHO. 1998.

23. Barker M, Grennwood K, Jackson M, Crowe S. Cognitive effects of long-term benzodiazepine use. CNS Drugs. 2004;18:37-48.

24. Dellaroza M. Projeto de assistência interdisciplinar ao idoso em atenção primária. Estação. 2005.

25. Verdoux $H$, Lagnaoui $R$, Begaud B. Is benzodiazepine use a risk factor for cognitive decline and dementia? A literature review of epidemiological studies. Psychol Med. 2005; 35:307-15.

26. Xavier F, Ferraz M, Argimon I, Moriguchi E. A relevância clínica da percepção subjetiva da perda de memória por pessoas de idade. Rev Bras Neurologia. 2001;37:24-8.

27. Argimon I. Desenvolvimento cognitivo na terceira idade [Tese] Porto Alegre: Pontifícia Universidade Católica do Rio Grande do Sul, Porto Alegre. 2002.

28. Lezak M. Neuropsychological assessment. 3. ed. New York: Oxford Universities Press. 1995.

29. Oliveira C, Souza C, Freitas T, Ribeiro C. Idosos e família: asilos ou casa. 2006 [acesso em 2007 Jan 04 jan. 2007]: Disponível em: http://www.psicologia.com.pt.

30. Argimon I, Stein L. Habilidades cognitivas em indivíduos muito idosos: um estudo longitudinal. Cad Saude Publica. 2005;21:64-72

31. Linden $M, B a r T$, Helmchen H. Prevalence and appopriateness of psychotropic drug use in old age: results from the Berlim Aging Study (BASE). Psychogeriatr. 2004; 6:461-80.

32. Janicak PG, Davis JM, Preskorn SH, Ayd J. FJ. Treatment with antiaanxiety and sedative hypnotic agents. In: Janicak PG,Janicak JM, Davis JM, Preskorn SH, Ayd Jr. FJ, Ayd Jr., FJ, Pavuluri M. editors. Principles and practice of psychopharmacotherapy. 3.ed. Lippincott: Williams \& Wilkins. 2001. p. 471-512.

33. Poyares D, Pinto L, Tavares S, Barros-Vieira S. Hipnoindutores e insônia. Rev Bras Psiquiatr. $2005 ; 27(1): 2-7$ 